\title{
Mechanism of Synaptic Dysfunction and How This Disruption in IGF-1 homeostasis Leads to Neurodegenerative Diseases: A Theory
}

Reagan $\mathrm{J}_{\text {Major }}{ }^{{ }^{*}}$ and Adrian A Jarquin-Valdivia ${ }^{2}$

${ }^{1}$ Department of Neurology, Washington University in St. Louis, St. Louis, MO 63130, United States of America

${ }^{2}$ TriStar Centennial Medical Center, Chief Neurology, Director Neurocritical Care, Medical Director Stroke Center, Director TeleNeurology, United States of America

"Corresponding author: Reagan Major, Department of Neurology, Washington University in St. Louis, St. Louis, MO 63130, United States of America, Tel: 314-362-5000; E-mail: ReaganJMajor@gmail.com

Received date: June 14, 2018; Accepted date: July 11, 2018; Published date: July 18, 2018

Copyright: (C) 2018 Major RJ, et al. This is an open-access article distributed under the terms of the Creative Commons Attribution License, which permits unrestricted use, distribution and reproduction in any medium, provided the original author and source are credited.

\section{Abstract}

Background: The diverse stimuli which ultimately trigger synaptic degeneration, may do so through a single, critical step or related pathways. The identification of such a step could possibly result in a significant breakthrough in our understanding of why synapses are particularly vulnerable. This could assist in the development of clinical strategies that protect synaptic function, possibly providing answers for a broad spectrum of disorders.

Methods: We propose that the methodology behind the mystery of synaptic disintegration is found in the 4dimensional aberrant neurological function. In the CNS this presents as delirium, in the PNS fragility (acquired hospital weakness).

Results: Clinically, we observe that the loss of synaptic function may occur faster than the recovery of the neurological circuit, causing a sort of accelerated aging. Further experiments will be required to gain important insight into the molecular mechanism of insulin-like growth factor 1 (IGF-1), its action on presynaptic and postsynaptic neurotransmitter release, and therapeutic ways to mediate this IGF-1 correlated age-associated decline. Further approaches to both observe and possibly correct these synaptic mechanisms include using ultrasound technology, electric stimulation of synaptic circuitry, radio wave, light stimulation, magnetic fields, virtual reality, and other physical methods that can interfere with the human's internal electrical system, stimulating systems at risk that have lost their synaptic pathways and resetting or preserving homeostatic clinical mechanisms and outcomes.

Conclusion: While there are a variety of clinically encountered conditions, such as sedatives, steroids, and immobility, that accelerate the synaptic dysfunction, we hypothesize the main critical pathway is that of Hebbian spike-timing-dependent plasticity (STDP), combining both the temporal element of neural circuitry and the dysregulation of the homeostatic functions that modulate synaptogenesis. The proposed 4D function of the nervous system relies on both timing and usage.

Keywords: Delirium; Encephalopathy; Synapse; Neurological dysfunction; Biomedical pathways; Biochemical mechanism; IGF; IGF-1; IGF-1 pathway; Ca++ homeostasis; Homeostasis; Electrical mechanism; Synaptic mechanism; Synaptic degeneration

\section{Introduction}

Previously, it might have seemed parsimonious to suggest that the diverse stimuli, which ultimately trigger synaptic degeneration, may do so through a single pathway or a critical series of closely related events. There are an estimated 100 billion neurons in the adult human brain, totaling around 0.15 quadrillion "at-risk" synapses [1]. The identification of such a step could possibly result in a significant breakthrough in our understanding of why synaptic units are particularly vulnerable and assist in the development of clinical strategies that protect synaptic function, perhaps providing answers for a broad spectrum of disorders. The methodology behind the synaptic disintegration is found in the difficult to study 4-dimensional (4D) aberrant neurological function. Clinically, we observe that the loss of synaptic function may occur faster than the recovery of the neurological circuit, causing a sort of accelerated aging. There are a variety of clinically encountered events, such as sedatives, benzodiazepines, steroids, and immobility that accelerates the synaptic dysfunction. We propose that by combining both the temporal element of neural circuitry and the deregulation of the homeostatic functions that modulate synaptogenesis and neuroplasticity, it is possible to pinpoint the mechanism behind many human degenerative diseases, predominantly neurodegenerative diseases.

\section{Substantiation}

Different mechanisms that occur during the neural pathway, such as patterns of interacting waves [2] low threshold $\mathrm{Na}+$ spikes and high threshold Ca+ spikes [3,4], postsynaptic N-methyl-D-aspartate receptors (NMDAR) and metabotropic glutamate receptors (mGluRs) impairment of mitochondrial transport [5-7], have been postulated to drive the specialized intercellular junction in which a nerve impulse passes to excitable cells, the synapse. Despite extensive research, the exact methodology that creates the synapse has remained elusive, possibly due to the diverse variety of synapse types, locations, uses, 
chronometry, age, state of the micromilieu, and because of multiple other underlying factors that have been thought to contribute to the neuronal junction.

Synaptic ontology seems based on the hierarchical description of synaptic structures and functions that underlie many biological processes. These functions are known play key roles in information processing in the nervous system, such as neurotransmission, confirming that synaptic declension is key to the initiation and progression of disease processes throughout the brain $[4,8]$. However to prevent synaptic decline, these plastic junctions are under biologically strict bidirectional homeostatic control. These homeostatic processes have also been thought to counteract the instability generated by Hebbian forces, adjusting synaptic strengths and intrinsic neuronal excitability, which keeps neural circuits functioning in biologically dynamic and heterogeneous ranges. When operating as expected, a precise time interval between the post and presynaptic spikes will determine the magnitude of the long-term potentiation or long-term depression of the synapse [3,9].

Mitochondria are highly dynamic organelles that divide, fuse, and move within axons and dendrites, mediating regulation of ion homeostasis, stress responses, cell survival and most significantly signal transduction [10]. Neuronal synapses are highly enriched with mitochondria in order to fulfill the high energy requirements needed to fuel the active processes required for synaptic transmission, producing the ATP needed to maintain synaptic ion homeostasis and phosphorylation reactions in presynaptic terminals [10-12]. This local ATP production by the mitochondria is imperative to maintaining synaptic transmission within the synapse, as mitochondria are essential for calcium homeostasis, a vital feature of the synapse $[7,13]$.

Changes in mitochondrial functions ( $\mathrm{Ca}+$ regulation, energy metabolism, and oxyradical production) play a crucial role in synaptic plasticity. Moreover, age-related cognitive impairment, and presumably the synaptic plasticity subservient learning and memory, is associated with structural abnormalities in mitochondria and oxidation of RNA and DNA $[10,14]$. In fact, a study by Kawamoto et al. has unveiled the importance of the specificity of $\mathrm{Ca}+$ in homeostatic and related signaling machinery. They postulated that $\mathrm{Ca}+$ levels are what underlies the unique responses to the same stimuli of different neurons, accounting for, at least partially, the selective impairment of neuronal subtypes and brain areas observed during aging and neurodegeneration [13]. Various studies have confirmed that calcium plays fundamental and diversified roles in neuronal physiology. For example, the regulation and the release of neurotransmitters from the presynaptic terminals influence both long-term potentiation and longterm depression [15-19]. Further, the increased $\mathrm{Ca}+$ levels in dendrites appear central to synaptic loss, destabilizing the cytoskeleton of dendritic spines, allowing them to involute. The impairment of the homeostatic mitochondrial transport system to both pre and postsynaptic terminals has been shown to cause synaptic loss, as the mitochondria are essential for Ca+ homeostasis [4]. Because $\mathrm{Ca}^{2+}$ is a fundamental signaling mechanism involved in almost all cellular physiological functions, subtle alterations of its homeostasis lead to profound functional change [13].

In addition to modulation of $\mathrm{Ca}+$ movements through the neuronal cellular compartments, an increasing number of signaling functions for mitochondria have recently been unveiled. For example, slow and prolonged changes in mitochondrial potential exhibit both temporal and spatial correlations with the intensity of electrical activity in glutamate-receptor-mediated patterned synaptic activity. Furthermore, impairment of mitochondrial transport into the presynaptic terminal has been shown to cause abnormal neurotransmission during intense stimulations [20], impaired presynaptic short-term plasticity, and accelerated synaptic depression under high-frequency firing [21]. Therefore it is possible that cognitive decline occurring with normal aging is not merely associated with significant neuronal loss, but is instead the result of changes in synaptic connectivity and loss of temporal regulation of homeostatic functions, particularly that of mitochondrial control of $\mathrm{Ca}+$.

While the dynamic mechanisms that underlie the degeneration of synapses throughout the neural circuit have remained elusive, the postulated defects in synaptic activity are associated with numerous neurological disorders [22], and as described above, the defects in homeostatic synaptic signaling could possibly contribute the disease pathogenesis of an array of neurological conditions. In fact, synapse loss is the strongest pathological correlate of dementia [23,24]. It has already been proven that dendritic spines of excitatory glutamatergic synapses, the most abundant type of synapse in the mammalian CNS, experience significant amounts of $\mathrm{Ca}+$ influx through $\mathrm{N}$-methyl-Daspartate (NMDA) receptors and voltage-dependent $\mathrm{Ca}+$ channels (VDCC), as well as release of Ca+ from the ER [25]. There is no question that the current scientific literature is on the dot with their postulation behind synaptogenesis. However, we believe those dots have not been connected because such studies and ideas have been limited to the medical technology currently available. However, the connection between these molecular methods of homeostasis and their correlation to synaptic regression lies in a dimension that has been left out of the literature: time.

\section{Methods/Theory}

When combining the molecular approaches discussed above with those of both temporal and Hebbian methodologies behind synapse, the once elusive dots become connected. Our proposed mechanism behind synaptogenesis incorporates the importance of the temporal axis on the strength of synaptic interactions between neurons in the brain and the subsequent deregulation of homeostatic mechanisms, allowing a $4 \mathrm{D}$ approach to synaptic plasticity. The Hebbian component of STDP implements the causal nature of Hebb's postulate: the strength of synapse whose activity leads to postsynaptic spikes and weakening synapses whose activity lags postsynaptic spikes, in other words "cell A reliably contributes to spiking of postsynaptic cell $\mathrm{B}$, the functional strength of the synapse from A to B is increased" [26]. The importance of the memo "cells that fire together, wire together" is imperative to the hypothesis of this paper, because the aberrant neurological function that occurs by virtue of synaptic deterioration leads to a deficit in synaptic function that occurs faster than its recovery, implying that interregional competition due to coupling between different neuronal dendritic regions leads to the potentiation of one population of synapses at the expense of another [27]. Furthermore, spike timing dependence originates in both molecular coincidence detection within classical long-term potentiation/Long-term depression (LTP/LTD) pathways (e.g., by NMDA receptors) and the temporal requirements for dendritic electrogenesis. Therefore, Hebbian STDP suggests that the unique benefits of spike timing dependence, while only one of many factors that oversee the plasticity of induction that influences synaptic activity, includes the incorporation of timing dependence, network stability, synaptic competition, sequence learning and prediction, all vital factors when analyzing the underlying mechanisms behind accelerated synaptic dysfunction [3]. 
In the brain, waves of electrical activity trace out distinct patterns across the nervous tissue. While it is unclear the exact patterns and roles that these waves play, traveling waves are highly correlated with the refinement of neural circuits, providing a suitable mechanism for mediating the refinement of synapses. Furthermore, the spatial scale of connectivity patterns is reliant on wave speed and STDP time constant, reinforcing the novel idea of the $4 \mathrm{D}$ component of the synaptic activity. Patterns of temporal differences between spike times of a given input neuron and output neuron can determine the strength of the respective synapse according to the Hebbian STDP rule, cluing us into the possible disintegration of synapses via the mechanism of accelerated aging that seems to occur during delirium associated immobility. Essential connectivity patterns can be unveiled using the wave theory to create a receptive field, determining largely the refined function of any given neuron. Disruptive patterns provide clues regarding the pathologies in the receptive field that can affect interneuronal correlations between synapses [2,28-30]. The model described by Bennett et al. [2] shows that the lateral interactions between synapses result directly from the timing dependence of STDP with is mapped onto the $4 \mathrm{D}$ conformation of neurological function via spatiotemporal correlations of traveling waves, further declaring that waves can induce strong correlations between any two input neurons irrespective of separation as long as they are correlated with a specific time lag $(\Delta \mathrm{x}=\mathrm{v} \Delta \mathrm{t})$. Clinically we can conclude that the temporal component of synaptic activity is imperative to correct functioning of the neural circuit. The challenge moving forward will be to understand how these mechanisms work together to integrate homeostatic information and orchestrate stable cellular, synaptic, and systemic functions in the neural circuit.

In this conceptual model, the electrical activity that the nervous system uses to establish stable communication throughout the recurrent network that drives LTP at synapses in the forward direction and LTD in the reverse, creates directional connections that are tuned, for example, due to selective responses, spontaneous repeated sequences of motor patterning, and the ability of the body to predict future events from past stimuli. In a clinical presentation, we hypothesize that the $4 \mathrm{D}$ degeneration of the synapses of neurotransmitter vesicles, due to exhaustion from over-stimulation, will present as rapid onset muscle weakness in the PNS (hospitalacquired weakness) and decline of cognitive ability (delirium) in the CNS. It has been suggested that stimulating activity may influence the rates of synaptic and neuronal degeneration both during normal aging and in neurodegenerative conditions in which cognitive decline or progressive impairment of muscle function is associated with early signs of synaptic dysfunction and demise [31-35]. In fact, recordings from muscles at miniature endplate potentials (mEEP) of patients with myasthenia gravis (MG), an autoimmune disorder that causes muscle weakness over time, had significantly reduced electrical amplitudes, which is interpreted as reduced postsynaptic excitability in the muscle [9]. High-frequency presynaptic firing is hypothesized to trigger LTP while low-frequency firing is hypothesized to drive LTD, pointing to the critical analysis that most synapses are found to be temporally correlated by presynaptic and postsynaptic depolarization. Further, simultaneous rapid sequential activation of two synaptically connected neurons leads to associative synaptic plasticity, or changes in the strength of synapses between them [3], driving the robust plasticity of cortical sensory maps that involve LTP and LTD, two main components of synaptic plasticity [36].

The use and disuse of synapses are thought to influence the progression of several neurodegenerative diseases in which synaptic degeneration is an early sign [37]. There is compelling evidence that some forms of synaptic remodeling or withdrawal are highly sensitive to activity. For instance, the rate of postnatal synapse elimination, a controlled process of presynaptic withdrawal that has been well characterized at developing neuromuscular junctions (NMJs) or following nerve regeneration in adults, is readily modifiable and strongly influenced by activity [37-39]. In an experiment conducted by Brown et al. [37], on the neuromuscular synapse reaction in various conditions on WldS mice concluded that intensive stimulation in vivo resulted complete nerve blockage in vivo and intensive stimulation ex vivo all reduced synaptic protection mediated by WldSgene expression, providing direct evidence that the rate of neuromuscular synaptic degeneration in response to axotomy is, in fact, sensitive to activity and that either complete disuse or sustained high-frequency stimulation can both cause motor nerve terminals more become vulnerable to potent triggers of degeneration. They further suggested that prolonged and intensive synaptic activity accelerates synaptic degeneration and the mechanism is sensitive to $\mathrm{Ca}+$. We interpret that the Hebbian STDP component of this synaptogenesis hypothesis provides the proven mechanism behind transneuronal degeneration, influencing the temporal element of neuronal firing, causing the progressive homeostatic decline.

Synaptic dysfunction and its demise are thought to influence the progression of several neurodegenerative diseases. The rates of synaptic and neuronal degeneration, which occur both during normal aging and in neurodegenerative conditions, contribute to cognitive decline or progressive impairment of muscle function [31,32,35,40,41]. The synaptic pruning mechanisms that occur during development, which allow for synapse elimination during postnatal refinement of neural circuits, have been found to contribute to synapse loss in Alzheimer's disease [4], suggesting that the disuse of such circuits causes them to stop firing. Spires-Jones [4] additionally states that impairment of mitochondrial transport to pre and postsynaptic terminals is thought to cause synapse loss and eventual dying-back of axons due to the essential roles of mitochondria in ATP production and calcium buffering further supporting our theory $[5,6]$.

While this is true for many neurological disorders, we will focus on how neuronal circuit malfunctioning explicitly causes delirium. Delirium is defined by the American Psychiatric Association's Diagnostic and Statistical Manual of Mental Disorders as a transient neurocognitive disorder, characterized by an acute onset and fluctuating course, inattention, cognitive dysfunction, and behavioral abnormalities, which develops in association with another underlying medical condition. It is possible that delirium causes motor fluctuations, such as myoclonus or tremulousness, due to the disruptors of key central neurotransmitters (for example, related to attentive and executive functions) leading to an inability in planning and sustaining movement pathways [42]. While current literature does not clearly define its cause, delirium has a consistent presentation that reflects dysfunction of a final common neural pathway [43-45] - or "all" pathways. One suggestion is an imbalance in the cholinergic and dopaminergic neurotransmitter systems [45], both commonly implicated in causing delirium, accounting for delirium symptoms and is also consistent with the malfunctioning of neuroanatomical pathways.

Delirium is defined by symptoms that expand far beyond an isolated mental disorder, with literature providing substantial evidence that it affects motor function as well. One highly studied decline of motor function, as a result of delirium, is the occurrence of frailty: a 
multidimensional loss of an individual's body system reserves which results in vulnerability to developing adverse health-related outcomes. Frailty is also defined by its effect on functional impairment, activity limitation, and participation restriction. Weakness often causes a multidimensional loss of an individual's body system reserves, resulting in vulnerability to developing sequential health-related outcomes. Some clinically reported indirect effects of fragility, due to the constraint of low physical activity, have presented as depressive symptoms and cognitive impairment, both consistent with the neurological symptoms of delirium [46]. Gupta et al. [45] state that a common neurological pathway may be responsible for specific 'core symptoms' of delirium (disorientation, cognitive deficits, sleep-wake cycle disturbance, disorganized thinking, and language abnormalities). They found that the prefrontal cortices, anterior and right thalamus, and right basilar mesial temporoparietal cortex may play a significant role in subserving delirium symptoms and maybe the 'final common pathway' for delirium from a variety of etiologies. Bellelli et al. [42] contribute that the physiological systems which are involved in the determination of frailty, including the brain, endocrine system, immune system, and skeletal muscle are mutually interrelated. Therefore, we conclude that the degeneration of the neural circuit responsible for the onset of delirium, may initiate a cascade of disruption of the temporal disruption of the mechanisms that drive the stimulation and communication of such areas in the brain, ultimately decreasing the strength and vitality of crucial synapses, leading to a multidimensional loss of body functions, a type of accelerated aging.

\section{Results}

Past theories have suggested that diverse arrays of mechanisms are responsible for synaptic degeneration. It has been noted that noted that the number of neurons in the brain does not appear to change significantly over our lifespan suggesting that the changes in the strength of connections between existing neurons could be the mechanism for memory formation $[47,48]$. We propose that this is also true for the causation of neurological disorders, specifically delirium and HAW. Some studies have used electroencephalography as a methodology of showing the diffuse slow-wave activity associated with delirium synaptic dysfunction [49], making it a possible clinical tool that can be used to support our theory, most importantly the wave propagation of neuronal firing discussed above. Studies have attempted to use light microscopy as a means of detecting the methods behind synaptic circuitry. However, the limitation of the z-axis and the fact that the resolution of light microscopy is generally larger than the size of an individual synapse make it less than ideal [4]. In addition, it explores tissue that is already dead, limiting the exploration of anatomy and not function. However, two-photon laser microscopy allows the visualization of synaptic features in vivo, allowing us to analyze and quantify the formation, elimination, and destabilization of axonal buttons dendritic spines and synapses [22]. However, these methods are error-prone, and have limitations but could still shed enough light as to guide, clarify and help formulate the next set of questions. Xie et al. [22] proposed an algorithm for automatic neuron reconstruction. The algorithm can handle complex structures adaptively and optimize the localization of bifurcations (a division of two branches), reconstructing the neuronal structures from confocal and multiphoton images. While the limitations of current technology may be the reason behind the elusivity of synaptic disintegration, recent trajectories show promising methods of detection in the near future.

\section{Discussion}

For the specificity of our theory, relating the loss of synaptic cohesion to delirium, it is necessary to diagnose and define delirium in the clinical setting. Symptom rating scales have certain advantages over pure clinical observation, specifically the objectivity, standardization, and ability to compare normative data. Some possibly useful screening scales include the Confusion Assessment Method [50], the Cognitive Test for Delirium (CTD) [51], and the Delirium Observation Scale [52]. The DRS revised version (DRS-R-98) is one of the most frequently used severity measure [43]. While there are other valid and reliable severity measures, these other scales have been criticized for their bias towards hyperactive detection of delirium symptomatology. Furthermore, the CTD, DRS-R-98, and DRS are the only tools that have been validated for their ability to distinguish delirium from dementia [45]. These clinical observation scales are helpful for our theory, allowing us to diagnose delirium and correlate the diagnosis with synaptic dysfunction.

\section{Application of biomedical theory for clinical implications: IGF homeostasis}

Clinically delirium is very common, especially in those of older age. Future protocols that prevent the decline of central and peripheral neurological synapses could improve the quality of life for many people and decrease medical costs relative to palliative care substantially. The disruption in IGF homeostasis due to the synaptic dysfunction causes some neurodegenerative diseases.

Numerous studies have discussed the importance of IGF-1 in neurological functioning as well as many other regulatory mechanisms. Systemic IGF-I can pass from the blood to the cerebrospinal fluid through the lipoprotein receptor-related protein 2 (LRP2), allowing it to cross the blood-brain-barrier by binding to the IGF-IR present on endothelial cells, where it is either picked up by astrocytes to be transferred to neurons or directly by neurons $[53,54]$. Therefore, IGF-I can act in the brain in an endocrine, paracrine or autocrine manner. The importance of IGF-1 on synaptogenesis has been thoroughly researched, concluding that the IGF-I/IGF-IR system regulates the proliferation and survival of neural progenitors, as well as the generation, differentiation, and maturation of neurons in multiple ways [55-57]. Therefore it is possible that IGF-I is part of the regulation of neuronal maturation, affecting axonal and dendritic growth, and establishing synapses in different brain areas independently of cell survival during synapse formation and in the adult CNS $[56,58,59]$. During development this has been demonstrated by it has been showing that IGF-I promotes the establishment of cerebellar synapses whereas lack of IGF-I facilitates its removal during postnatal development [60]. Furthermore the CNS phenotype of IGF-1 knockout mice. IGF-1 gene disruption results in reduced brain size, CNS hypomyelination, a decrease in dendritic spine density, reduction in presynaptic protein- synaptotagmin levels and loss of hippocampal granule and striatal parvalbumin-containing neurons, implying synapse loss in the absence of IGF-1 [61]. Further, Carson [62] showed that an increase in IGF-1 results in a larger brain as well as increased myelination of the CNS, in transgenic mice.

Therefore, one could postulate that calcium is essential to the homeostatic regulation of IGF-1 synaptogenesis. When levels of $\mathrm{Ca}+$ are sufficiently elevated during rapid neuronal firing, the calcium signaling activates the transcription of IGF binding proteins [63] as well as releases IGF-1 is released via exocytosis, further modifying 
IGF-1 signaling [64]. In other words, IGF-1 is secreted by an activitydependent, Ca2+-regulated vesicular pathway of exocytosis [59]. The importance of this paper is to recognize not only the Hebbian component of $\mathrm{Ca}+$ depolarization in synaptogenesis but the novel concept of $4 \mathrm{D}$ neurological functions too.

If the temporal basis of $\mathrm{Ca}+$ depolarization is aberrant, then it is possible that the mechanism of IGF-1 release becomes anomalous as well. While multiple factors can result in an excitatory postsynaptic potential, opening probability (mean open dwell) time, is a significant factor in triggering $\mathrm{Ca}+$ influx through the postsynaptic NMDA receptors, resulting in subsequent depolarization. Deak et al. [64] state that $10-20 \mathrm{~ms}$ is the critical time window in which the stimulus must reach the depolarized postsynaptic cell. Regulation of IGF-1 excretion is key, however, in turn, IGF-I promotes adult neurogenesis by also regulating NSC number and differentiation, directing neuronal positioning and migration [59], demonstrating the importance of timing in the relation of both regulatory mechanisms.

The timing of $\mathrm{Ca}+$ is important to the correct homeostatic regulation of IGF-1 because the influx of $\mathrm{Ca}+$ through the postsynaptic NMDA receptors leads to the excretion of this vital hormone. Cognitive deficits associated with aging have found to relate to changes in NMDA receptor subunit expression. Most importantly these deficits are age-dependent changes reversed by systematic IGF-1 treatment, suggesting that the effects of IGF-1 on cognitive function can be mediated, at least in part by modulation of synaptic function in general, and more specifically NMDA receptors $[65,66]$. IGF-1 is one of only a few interventions that have consistently been reported to reverse cognitive decline with age. Therefore, further research in this area will hopefully identify targets for selective therapeutic interventions that improve cognitive function in the elderly patients. The theory of the Hebbian STDP mechanism behind synaptic pathways lets us explore future mediation of synaptic dysfunction, specifically the synaptic pathway of the IGF-1 hormone, possibly mediating a wide array of diseases.

The muscles, brain, bones, and skin are affected by the lack of IGF-I, as reflected by the muscle hypoplasia and the reduced brain size, ossification, and skin thickness found in the KO mice [61,67]. The importance of this observation lies in the establishment that IGF- 1 and its signaling pathway have an important role in the cognitive decline in aged participants [59]. Furthermore, physical exercise enhances IGF-I entry into the brain $[54,68,69]$, which proves that disuse of skeletal muscle may lead to hospital-acquired weakness (HAW). Evidence supports this theory by indicating that IGF-I promotes cell survival by inhibiting apoptosis both in vivo and in vitro, observed in neural progenitors and multiple neuronal types such as cortical cells, motoneurons, Purkinje cells, and optic neural progenitor cells [70,71]. Aleman et al. reported that IGF-1 levels in participants 65-76 years of age were associated with perceptual-motor performance and information processing speed. In a 3-year longitudinal study, Deak et al. [64] found an association between low levels of IGF-1 and reduced processing speed, which would explain age-related delirium. These conclusions indicate an important association between cognitive function, motor function, and IGF-1 levels in older adults, possibly suggesting that restoring synaptogenesis via IGF-1 may prove a successful strategy for reversing age-related synaptic dysfunction and ameliorate cognitive and physical malfunction.

\section{Conclusion}

While is likely that minor defects in multiple processes, accumulate during aging and thus add to the complexity of synaptic dysfunction and cognitive decline in the individual patient, the pathway of IGF-1 plays an important role in synaptogenesis. The overarching conclusion from numerous studies is that IGF-1 deficiency is an essential contributing factor in cognitive impairment both in adults and aged humans as well as rodent models of aging. IGF-1, among other things, is considered an important neurotrophic hormone, indicating a correlation between a decrease in circulating levels of IGF-1 with age and synaptic dysfunction.

Future protocols may find ways to induce the firing of synaptic junctions, causing the mechanism behind IGF-1 excretion to potentiate despite the aging body's internal degrading synaptogenesis. IGF-1 is produced in the brain IGF-1 production in the brain is not only a response to neuronal injury (ischemia or focal brain injury), but it is also dependent on neuronal activity. Therefore, by artificially stimulating the body's response to neuronal damage or by stimulating synaptogenesis, one should be able to induce the production of IGF-1. Further, the effects of IGF-1 on inflammatory responses is beyond the scope of this paper; however, it is noteworthy that recent findings support a role of IGF-1 treatment in protecting synaptic plasticity from inflammatory agent, possibly suggesting that stimulating an artificial inflammatory response in the brain would induce the body to produce IGF-1, a response to this falsified neuronal injury. The use of a monoclonal antibody that stimulates the immune response of specific neuronal receptors; or the insertion of an IGF-1 chemical derivative that is attached to a monoclonal antibody for the IGF-1 receptors in neuronal cells, stimulating the receptors to trigger functions similar to IGF-1 and overcoming the age-related synaptic decline and related dysregulation of homeostasis. Regardless, a combination of stimulatory and regulatory solutions must be enacted, because the proposed $4 \mathrm{D}$ function of the nervous system relies on both timing and usage.

Further experiments will be required to gain important insight into the molecular mechanism of IGF-1, its action on presynaptic and postsynaptic neurotransmitter release, and therapeutic ways to mediate this IGF-1 correlated age-associated decline. Further approaches to both observe and possibly correct these synaptic mechanisms include using ultrasound technology, electric stimulation of synaptic circuitry, radio wave, light stimulation, magnetic fields, virtual reality, and other physical methods that can interfere with the human's internal electrical system, stimulating systems at risk that have lost their synaptic pathways and resetting or preserving homeostatic clinical mechanisms and outcomes.

\section{References}

1. von Bartheld CS, Bahney J, Herculano-Houzel S (2016) The search for true numbers of neurons and glial cells in the human brain: A review of 150 years of cell counting. J Comp Neurol 524: 3865-3895.

2. Bennett J, Bair W (2015) Refinement and Pattern Formation in Neural Circuits by the Interaction of Traveling Waves with Spike-Timing Dependent Plasticity. PLoS Comput Biol 11: e1004422.

3. Feldman DE (2012) The spike-timing dependence of plasticity. Neuron 75: 556-571.

4. Spires-Jones T, Hyman B (2014) The Intersection of Amyloid Beta and Tau at Synapses in Alzheimer's Disease. Neuron 82: 756-771.

5. Kopeikina KJ, Polydoro M, Tai HC, Yaeger E, Carlson GA, et al. (2013) Synaptic alterations in the rTg 4510 mouse model of tauopathy. J Comp Neurol 521: 1334-1353. 
6. Sheng ZH, Cai Q (2012) Mitochondrial transport in neurons: impact on synaptic homeostasis and neurodegeneration. Nat Rev Neurosci 13: 77-93.

7. Eckert A, Nisbet R, Grimm A, Götz J (2014) March separate, strike together - Role of phosphorylated TAU in mitochondrial dysfunction in Alzheimer's disease. Biochim Biophys Acta 1842: 1258-1266.

8. Zhang W, Zhang Y, Zheng H, Zhang C, Xiong W, et al. (2007) SynDB: a Synapse protein DataBase based on synapse ontology. Nucleic Acids Res 35(Database): D737-D741.

9. Wondolowski J, Dickman D (2013) Emerging links between homeostatic synaptic plasticity and neurological disease. Front Cell Neurosci 7: 223.

10. Mattson M, Gleichmann M, Cheng A (2008) Mitochondria in Neuroplasticity and Neurological Disorders. Neuron 60: 748-766.

11. David G, Barrett EF (2000) Stimulation-evoked increases in cytosolic $[\mathrm{Ca}(2+)]$ in mouse motor nerve terminals are limited by mitochondrial uptake and are temperature-dependent. J Neurosci 20: 7290-7296.

12. Tsang CW, Elrick DB, Charlton MP (2000) alpha-Latrotoxin releases calcium in frog motor nerve terminals. J Neurosci 20: 8685-8692.

13. Kawamoto EM, Vivar C, Camandola S (2012) Physiology and Pathology of Calcium Signaling in the Brain. Frontiers in Pharmacology 3: 61 .

14. Liu J, Head E, Gharib AM, Yuan W, Ingersoll RT, et al. (2002) Memory loss in old rats is associated with brain mitochondrial decay and RNA/DNA oxidation: Partial reversal by feeding acetyl-L-carnitine and/or R- -lipoic acid. Proc Natl Acad Sci USA 99: 2356-2361.

15. Grover LM, Teyler TJ (1990) Two Components of Long-term Potentiation Induced by Different Patterns of Afferent Activation. Nature 347: 477-479.

16. Impey S, Mark M, Villacres EC, Poser S, Chavkin C, et al. (1996) Induction of CRE-Mediated Gene Expression by Stimuli That Generate Long-Lasting LTP in Area CA1 of the Hippocampus. Neuron 16: 973-982.

17. Bolshakov VY, Siegelbaum SA (1994) Postsynaptic induction and presynaptic expression of hippocampal long-term depression. Science 264: 1148-1152.

18. Christie BR (1996) Long-term Depression (LTD) in the Hippocampus. Hippocampus 6: 1-2.

19. Christie B.R, Magee JC, Johnston D (1996) Dendritic calcium channels and hippocampal long-term depression. Hippocampus 6: 17-23.

20. Verstreken P, Ly CV, Venken KJ, Koh TW, Zhou Y, et al. (2005) Synaptic mitochondria are critical form mobilization of reserve pool vesicles at Drosophila neuromuscular junctions. Neuron 47: 365-378.

21. Ma H, Cai Q, Lu W, Sheng ZH, Mochida S (2009) KIF5B motor adaptor syntabulin maintains synaptic transmission in sympathetic neurons. Neurosci 41: 13019-13029.

22. Xie Q, Chen X, Deng H, Liu H, Sun Y, et al. (2017) An automated pipeline for bouton, spine, and synapse detection of in vivo two-photon images. BioData Mining 10: 40.

23. DeKosky S, Scheff S (1990) Synapse loss in frontal cortex biopsies in Alzheimer's disease: correlation with cognitive severity. Annals of Neurol 27: 457-464.

24. DeKosky ST, Scheff SW, Styren SD (1996) Structural correlates of cognition in dementia: quantification and assessment of synapse change. Neurodegeneration 5: 417-421.

25. Toresson H, Grant SG (2005) Dynamic distribution of endoplasmic reticulum in hippocampal neuron dendritic spines. Eur J Neurosci 22: 1793-1798.

26. Morris RG (1999) D.O. Hebb: The Organization of Behavior, Wiley: New York; 1949. Brain Res Bull 50: 437.

27. Bar Ilan L, Gidon A, Segev I (2011) Interregional synaptic competition in neurons with multiple STDP-inducing signals. J Neurophysiol 105: 989-998.

28. Markram H, Lübke J, Frotscher M, Sakmann B (1997) Regulation of synaptic efficacy by coincidence of postsynaptic APs and EPSPs. Science 275: 213-215.
29. Zhang LI, Tao HW, Holt CE, Harris WA, Poo M (1998) A critical window for cooperation and competition among developing retinotectal synapses. Nature 395: 37-44.

30. Caporale N, Dan Y (2008) Spike Timing-Dependent Plasticity: A Hebbian Learning Rule. Annu Rev Neurosci 31: 25-46.

31. Valdez G, Tapia JC, Kang H, Clemenson GD Jr, Gage FH, et al. (2010) Attenuation of age-related changes in mouse neuromuscular synapses by caloric restriction and exercise. Proc Natl Acad Sci U S A 107: 14863-14868.

32. Li Y, Lee Y, Thompson WJ (2011) Changes in aging mouse neuromuscular junctions are explained by degeneration and regeneration of muscle fiber segments at the synapse. J Neurosci 31: 14910-14919.

33. Punga AR, Ruegg MA (2012) Signaling and aging at the neuromuscular synapse: lessons learnt from neuromuscular diseases. Curr Opin Pharmacol 12: 340-346.

34. Stern Y (2012) Cognitive reserve in ageing and Alzheimer's disease. Lancet Neurol 11: 1006-1012.

35. Shors TJ, Anderson ML, Curlik DM 2nd, Nokia MS (2012) Use it or lose it: how neurogenesis keeps the brain fit for learning. Behav Brain Res 227: 450-458.

36. Feldman DE (2009) Synaptic mechanisms for plasticity in neocortex. Annu Rev Neurosci 32: 33-55.

37. Brown R, Hynes-Allen A, Swan A, Dissanayake K, Gillingwater T, et al. (2015) Activity-dependent degeneration of axotomized neuromuscular synapses in WldS mice. Neuroscience 290: 300-320.

38. Walsh MK, Lichtman JW (2003) In vivo time-lapse imaging of synaptic takeover associated with naturally occurring synapse elimination. Neuron 37: 67-73.

39. Caroni P, Chowdhury A, Lahr M (2014) Synapse rearrangements upon learning: from divergent-sparse connectivity to dedicated sub-circuits. Trends Neurosci 37: 604-614

40. Fischer LR, Culver DG, Tennant P, Davis AA, Wang M, et al. (2004) Amyotrophic lateral sclerosis is a distal axonopathy: evidence in mice and man. Exp Neurol 185: 232-240.

41. Frick KM, Benoit JD (2010) Use it or lose it: environmental enrichment as a means to promote successful cognitive aging. ScientificWorldJournal 10: 1129-1141.

42. Bellelli G, Moresco R, Panina-Bordignon P, Arosio B, Gelfi C, et al. (2017) Is Delirium the Cognitive Harbinger of Frailty in Older Adults? A Review about the Existing Evidence. Front Med 4: 188.

43. Trzepacz PT, Mittal D, Torres R, Kanary K, Norton J (2001) Validation of the Delirium Rating Scale-revised-98: comparison with the delirium rating scale and the cognitive test for delirium. J Neuropsychiatry Clin Neurosci 13: 229-242.

44. Trzepacz P (1999) Update on the Neuropathogenesis of Delirium. Dementia and Geriatric Cognitive Disorders 10: 330-334.

45. Gupta N, de Jonghe J, Schieveld J, Leonard M, Meagher D (2008) Delirium phenomenology: What can we learn from the symptoms of delirium?. J Psychosom Res 65: 215-222.

46. Ding Y, Kuha J, Murphy M (2017) Pathways from physical frailty to activity limitation in older people: Identifying moderators and mediators in the English Longitudinal Study of Ageing. Exp Gerontol 98: 169-176.

47. Cajal SRY (1894) The Croonian Lecture: La fine structure des centres nerveux. Proceedings of the Royal Society of London 55: 444-468.

48. Jones EG (1994) Santiago Ramon y Cajal and the croonian lecture, March 1894. Trends in Neurosciences 17: 190-192.

49. Plaschke K, Hill H, Engelhardt R, Thomas C, von Haken R, et al. (2007) EEG changes and serum anticholinergic activity measured in patients with delirium in the intensive care unit. Anaesthesia 62: 1217-1223.

50. Inouye S (1990) Clarifying Confusion: The Confusion Assessment Method. Annals of Internal Medicine 113: 941

51. Hart RP, Levenson JL, Sessler CN, Best AM, Schwartz SM, et al. (1996) Validation of a cognitive test for delirium in medical ICU patients. Psychosomatics 37: 533-546. 
Citation: Major RJ, Jarquin-Valdivia AA (2018) Mechanism of Synaptic Dysfunction and How This Disruption in IGF-1 homeostasis Leads to Neurodegenerative Diseases: A Theory. Biol Med (Aligarh) 10: 445. doi:10.4172/0974-8369.1000445

Page 7 of 7

52. Schuurmans MJ, Shortridge-Baggett LM, Duursma SA (2003) The Delirium Observation Screening Scale: a screening instrument for delirium. Res Theory Nurs Pract 17: 31-50.

53. Nishijima T, Piriz J, Duflot S, Fernandez AM, Gaitan G, et al. (2010) Neuronal Activity Drives Localized Blood-Brain-Barrier Transport of Serum Insulin-like Growth Factor-I into the CNS. Neuron 67: 834-846.

54. Fernandez AM, Torres-Aleman I (2012) The Many Faces of Insulin-like Peptide Signalling in the Brain. Nat Rev Neurosci 13: 225-239.

55. Cheng CM, Cohen M, Tseng V, Bondy CA (2001) Endogenous IGF1 Enhances Cell Survival in the Postnatal Dentate Gyrus. J Neurosci Res 64: 341-347.

56. O'Kusky JR, Ye P, D'Ercole AJ (2000) Insulin-like Growth factor-I Promotes Neurogenesis and Synaptogenesis in the Hippocampal Dentate Gyrus During Postnatal Development. J Neurosci 20: 8435-8442.

57. Chaker Z, Aid S, Berry H, Holzenberger M (2015) Suppression of IGF-I Signals in Neural Stem Cells Enhances Neurogenesis and Olfactory Function During Aging. Aging Cell 14: 847-856.

58. Cao P, Maximov A, Südhof TC (2011) Activity-Dependent IGF-1 Exocytosis Is Controlled by the Ca2+-Sensor Synaptotagmin-10. Cell 145: 300-311.

59. Nieto-Estevez V, Defterali C, Vicario-Abejon C (2016) IGF-I: A Key Growth Factor That Regulates Neurogenesis and Synaptogenesis from Embryonic to Adult Stages of the Brain. Frontiers in Neuroscience 10: 52.

60. Kakizawa S, Yamada K, Iino M, Watanabe M, Kano M (2003) Effects of Insulin-like Growth Factor I on Climbing Fibre Synapse Elimination During Cerebellar Development: Effects of IGF-I on Climbing Fibre Synapses. European Journal of Neuroscience 17: 545-554.

61. Beck KD, Powell-Braxton L, Widmer HR, Valverde J, Hefti F (1995) Igf1 Gene Disruption Results in Reduced Brain Size, CNS Hypomyelination, and Loss of Hippocampal Granule and Striatal Parvalbumin-containing Neurons. Neuron 14: 717-730.

62. Carson MJ, Behringer RR, Brinster RL, McMorris FA McMorris (1993) Insulin-like Growth Factor I Increases Brain Growth and Central Nervous System Myelination in Transgenic Mice. Neuron 10: 729-740.
63. Zhang SJ, Zou M, Lu L, Lau D, Ditzel DA, et al. (2009) Nuclear Calcium Signaling Controls Expression of a Large Gene Pool: Identification of a Gene Program for Acquired Neuroprotection Induced by Synaptic Activity. PLoS Genetics 5: e1000604.

64. Deak F, Sonntag WE (2012) Aging, Synaptic Dysfunction, and InsulinLike Growth Factor (IGF)-1. J Gerontol A Biol Sci Med Sci 67A: 611-625.

65. Sonntag WE, Bennett SA, Khan AS, Thornton PL, Xu X, et al. (2000) Age and Insulin-like Growth Factor-1 Modulate N-methyl-D-aspartate Receptor Subtype Expression in Rats. Brain Res Bull 51: 331-338.

66. Sonntag WE, Ramsey M, Carter CS (2005) Growth Hormone and Insulin-like Growth Factor-1 (IGF-1) and Their Influence on Cognitive Aging. Ageing Res Rev 4: 195-212.

67. Pichel JG, Fernández-Moreno C, Vicario-Abejón C, Testillano PS, Patterson PH, et al. (2003) Developmental Cooperation of Leukemia Inhibitory Factor and Insulin-like Growth Factor I in Mice Is Tissuespecific and Essential for Lung Maturation Involving the Transcription Factors Sp3 and TTF-1. Mechanisms of Development 120: 349-361.

68. Trejo JL, Carro E, Torres-Aleman I (2001) Circulating Insulin-like Growth Factor I Mediates Exercise-induced Increases in the Number of New Neurons in the Adult Hippocampus. J Neurosci 21: 1628-1634.

69. Glasper ER, Llorens-Martin MV, Leuner B, Gould E, Trejo JL (2009) Blockade of Insulin-like Growth factor-I Has Complex Effects on Structural Plasticity in the Hippocampus. Hippocampus 20: 706-712.

70. Gago N, Avellana-Adalid V, Baron-Van Evercooren A, Schumacher M (2003) Control of Cell Survival and Proliferation of Postnatal PSANCAM+ Progenitors. Molecular and Cellular Neuroscience 22: 162-178.

71. Croci L, Barili V, Chia D, Massimino L, van Vugt R, et al. (2011) Local Insulin-like Growth Factor I Expression Is Essential for Purkinje Neuron Survival at Birth. Cell Death \& Differentiation 18: 48-59. 